\title{
IMMEDIATE BREAST RECONSTRUCTION WITH DEFINITIVE PRE-PECTORAL PROSTHESIS AFTER NIPPLE SPARING MASTECTOMY THROUGH INFRAMAMMARY FOLD WITHOUT USING AN ACELLULAR MATRIX: RESULT OF 130 CASES
}

Alessandra Amatuzi Cordeiro Fornazari¹, Leonardo Paese Nissen¹, Flavia Kuroda¹, Maíra Teixeira Dória, Cícero de Andrade Urban ${ }^{1}$

1Oncoclínica - Curitiba (PR), Brazil.

Introduction: Breast reconstruction with prosthesis after mastectomy is currently the main reconstruction technique used in the world. The pre-pectoral prosthesis is progressively being more used, and has advantages in relation to the submuscular prosthesis, such as less pain in the postoperative period, decreased muscular deficit, breast animation, better aesthetic result, besides reducing the time of surgical morbidity, for being easier to execute. Objectives: To review the results and complications of patients submitted to breast reconstruction with definitive prosthesis without using an acellular matrix (ADM). Methods: Retrospective study including patients who underwent immediate reconstruction with pre-pectoral definitive prosthesis after nipple sparing mastectomy (NSM), with incision through the inframammary fold (IMD), without using ADM, between January, 2018, and July, 2019. We collected demographic data, types of therapy and surgical data. The complications and secondary surgical interventions were also assessed. The Fisher's Exact Test was used for statistical analysis (p lower than 0.05). Results: During the study period, 130 reconstructions were performed in 87 patients. Mean age was 43 years. Thirty two mastectomy procedures (24.6\%) presented at least one complication, and the most common ones were: flap necrosis $(\mathrm{N}=13)$, persistent seroma $(\mathrm{N}=10)$, and prosthesis exposure $(\mathrm{N}=9)$. Of this total, 21 were submitted to a new surgical procedure, and $12(9.2 \%)$ evolved with loss of prosthesis in an average of 64 days (12 to 180 days) after the first surgery. The main risk factors associated with loss of prosthesis were smoking (OR 4; 1.48-10.8) and BMI higher than 25 (OR 4.4; 1.24-15.6), both with statistical significance $(\mathrm{p}<0.05)$. The presence of previous radiotherapy $(42.8 \%$ x $21.5 \%)$ or adjuvant therapy (37.5\% x 21.5\%) and diabetes mellitus ( $42.9 \%$ x $23.6 \%$ ) show a tendency to higher chances of complications, however, these were not statistically significant. The other assessed factors did not present correlation with the complications. Regarding the late aesthetic result, we only assessed the patients who were followed up for more than 6 months. Of the 52 breasts, $69.3 \%$ did not present capsular contracture, and $28.8 \%$ presented contracture in Grades I or II of the Baker scale. Rippling was identified in only 13 breasts (25\%). There was no prosthesis dislocation or breast animation. Conclusion: Breast reconstruction with definitive pre-pectoral prosthesis after NSM using IMD is a promising, safe and economically advantageous technique, presenting results and complications similar to the cases that use ADM or place the retromuscular prosthesis. 